
\title{
25 Research Soure \\ YTHDC2 Promotes the Apoptosis of Colorectal Cancer Cells Through the p38MAPK Signaling Pathway
}

\section{Tao Yu}

Tianjin Medical University General Hospital

\section{YanYan Xu}

Second Affiliated Hospital of Anhui Medical University

\section{Tao Sun}

\author{
Nankai University
}

Di Huang

Tianjin Medical University General Hospital

\section{Feng Cao}

Second Affiliated Hospital of Anhui Medical University

\section{Xin Gao}

Tianjin Medical University General Hospital

\section{Gang Liu ( $\nabla$ lg1059@tmu.edu.cn )}

Tianjin Medical University General Hospital https://orcid.org/0000-0001-8037-0850

\section{Primary research}

Keywords: YTH-domain containing 2; N6-methyladenosine; Colorectal cancer; Post-transcriptional modification; Apoptosis

Posted Date: May 18th, 2020

DOI: https://doi.org/10.21203/rs.3.rs-27416/v1

License: (a) (1) This work is licensed under a Creative Commons Attribution 4.0 International License. Read Full License 


\section{Abstract \\ Background}

The role of the $\mathrm{m}^{6} \mathrm{~A}$-binding protein YTHDC2 in the occurrence and development of colorectal cancer (CRC) is unclear. We aimed to explore the molecular mechanisms underlying this process and clarify the signaling pathway involved.

\section{Methods}

Firstly, the relationship between YTHDC2 and CRC in TCGA database was analyzed to identify relevant signaling pathways and biological processes. Then, western blot was used to analyze expression of YTHDC2 in HCT116 and Caco2 cells. After knockdown or overexpression of YTHDC2 in the above cells, RT-PCR and western blot were used to analyze p38MAPK, p-p38MAPK, and downstream apoptosis-related proteins in the MAPK signaling pathway. Flow cytometry was performed to detect changes in apoptosis.

\section{Results}

And the results were shown that the expression of YTHDC2 was significantly lower in tumor tissues than in normal tissues. Increased expression of YTHDC2 was associated with better overall survival among patients with CRC. Gene set enrichment analysis revealed that YTHDC2 regulates the MAPK signaling pathway. Flow cytometry revealed apoptosis was significantly reduced and enhanced in response to YTHDC2 knockdown and overexpression, respectively. There was no significant change in the expression of p38MAPK, while p-p38MAPK was significantly increased in response to overexpression and decreased in response to knockdown. Gene Expression Profiling Interactive Analysis showed apoptotic protein expression to be positively correlated with YTHDC2 expression, consistent with the results of RT-PCR and western blot.

\section{Conclusion}

In general, apoptosis of CRC cells is promoted by YTHDC2 via activation of the exogenous death receptor and endogenous mitochondrial apoptosis-related pathways in the p38MAPK signaling pathway.

\section{Background}

Colorectal cancer (CRC) accounted for $6.1 \%$ of all cancers worldwide in 2018, with a mortality rate of $9.2 \%$, which was second only to lung cancer ${ }^{[1]}$. Temporal profiles and demographic projections indicate that the global burden of CRC is likely to increase by $60 \%$ by $2030^{[2]}$. In China particularly, the incidence and mortality of CRC have increased in recent decades. A large number of studies have the development 
and metastasis of CRC to occur via complex processes involving multiple genes. Therefore, the identification of genetic and molecular markers related to the development of CRC, as well as the study of their association with the pathogenesis and biological characteristics, will enable evaluation of the prognosis of CRC.

The $\mathrm{N}^{6}$-methyladenosin $\left(\mathrm{m}^{6} \mathrm{~A}\right)$ modification is the most common post-transcriptional modification of eukaryotic RNA ${ }^{[3]}$. This modification occurs in a well-defined RNA motif, DRACH (D can be A, C or U; R can be either $\mathrm{A}$ or $\mathrm{G}$; and $\mathrm{H}$ can be $\mathrm{A}, \mathrm{C}$ or $\mathrm{U})^{[4]}$. The formation of the $\mathrm{m}^{6} \mathrm{~A}$ modification has been demonstrated to be a dynamic process, and its function is determined by "Writer", "Eraser", and "Reader" enzymes. Writer, also referred to as the methyltransferase complex, is composed of components include methyltransferase-like 3 (METTL3) ${ }^{[5]}$, METTL14 ${ }^{[6]}$, and WTAP ${ }^{[7]}$. Eraser refers to demethylases, including ALKBH5 ${ }^{[8]}$ and $\mathrm{FTO}^{[9]}$, and Reader-also called $\mathrm{m}^{6} \mathrm{~A}$-binding protein-recognizes the $\mathrm{m}^{6} \mathrm{~A}$ modification. Currently, the $\mathrm{m}^{6} \mathrm{~A}$-binding proteins include members of the YTH-domain protein family (YTHDF1, YTHDF2, YTHDF3, YTHDC1, and YTHDC2 $)^{[10]}$ and the heterogeneous nuclear ribonucleoprotein (HNRNP) family (HNRNPA2B1 and HNRNPC) ${ }^{[11]}$. By regulating the stability, shear, transport, localization, and translation of RNA at the post-transcriptional level, the $\mathrm{m}^{6} \mathrm{~A}$ plays a key role in the dynamic regulation of cell stemness, differentiation, and energy balance.

The evolutionarily conserved YTH domain contained in the YTH protein family can selectively recognizes $\mathrm{m}^{6} \mathrm{~A}^{[12]}$. In the cytoplasm, YTHDF3 promotes protein synthesis in synergy with YTHDF1 ${ }^{[13]}$.

Destabilization of $\mathrm{m}^{6} \mathrm{~A}$-containing RNA is carried out by YTHDF2 through direct recruitment of the carbon catabolite repressor 4-negative on TATA (CCR4-NOT) deadenylase complex, which accelerates mRNA degradation ${ }^{[14]}$. In the nucleus, YTHDC1 regulates the splicing of target $m R N A^{[15]}$. The biological function of the final member of the YTH protein family, YTHDC2, is still not fully understood. A study by Kretschmer et al. ${ }^{[16]}$ demonstrated that YTHDC2 is mainly enriched in the perinuclear space and interacts with the small ribosomal subunit to improve the efficiency of translation. Hsu et al. ${ }^{[4]}$ reported the translation efficiency of the target gene Smc3 to be decreased by $37 \%$ in the testicular tissue of YTHDC2knockout mice. Other studies have shown that the recognition of $m^{6} \mathrm{~A}$ by YTHDC2 is crucial for embryonic differentiation ${ }^{[17,18]}$. Furthermore, $Y T H D C 2$ may be a susceptibility gene for pancreatic cancer ${ }^{[19]}$ and plays an important role in the development of liver cancer ${ }^{[20]}$. However, there has been little research into the relationship between YTHDC2 and CRC.

The present research aimed to study the role of the $\mathrm{m}^{6} \mathrm{~A}$-binding protein $\mathrm{YTHDC2}$ in $\mathrm{CRC}$, explore the underlying molecular regulatory mechanism, and clarify the signaling pathway involved. The purpose of this was to elucidate comprehensive information regarding the important role of this protein in the occurrence and development of CRC. The results presented here may provide a new direction for the development of drug treatment for CRC.

\section{Methods}




\section{Antibodies and plasmids}

The Anti-YTHDC2 (ab220160), Anti-p38MAPK (ab31828), Anti-p-p38MAPK (ab4822), Anti-Fas (ab82419), Anti-Caspase-8 (ab25901), Anti-Caspase-9 (ab32539), Anti-Bax (ab32503), Anti-Cytochrome C (ab13575), and Anti-Glyceraldehyde 3-phosphate dehydrogenase (Anti-GAPDH; ab8245) antibodies were purchased from Abcam (USA); Anti-Fas Ligand (bs-0216R) and Anti-Caspase-12 (bs-0967R) were purchased from Bioss (Beijing, China); Horseradish peroxidase (HRP)-conjugated secondary antibodies were purchased from Cell Signaling Technology (CST, USA). Fluorescein isothiocyanate (FITC)- and tetramethylrhodamine (TRITC)-labeled secondary antibodies were purchased from Earthox LLC. (USA). The pCMV3-YTHDC2Flag and pCMV3-C-Flag plasmids were synthesized by Sino Biological (SB, Beijing, China), and siYTHDC2 was synthesized by GenePharma (Suzhou, China).

\section{Cell culture and plasmid transfection}

The HCT116 and Caco2 cell lines were purchased from the American Type Culture Collection (ATCC) and cultured in Dulbecco's Modified Eagle Medium (HyClone, USA) with $10 \%$ fetal bovine serum (Thermo Fisher Scientific, USA), streptomycin $(100 \mu \mathrm{g} / \mathrm{mL})$ and penicillin (100 units $/ \mathrm{mL})$ at $37^{\circ} \mathrm{C}$ in a saturated humidity atmosphere containing $5 \% \mathrm{CO}_{2}$. When the cell density reached $80 \%-90 \%$, they were divided into four groups according to the subsequent treatment: the YTHDC2 group was transfected with the pCMV3YTHDC2-Flag plasmid, the siYTHDC2 group was transfected with si-YTHDC2, the con-YTHDC2 group was transfected with pCMV3-C-Flag, and the NC-siYTHDC2 group was transfected with si-NC as a control group. Transfection with plasmids and siRNA was carried out using the Lipofectamine ${ }^{\mathrm{TM}} 2000$ reagent (Invitrogen, USA).

\section{Bioinformatics data analysis}

The relationship between CRC and YTHDC2 in The Cancer Genome Atlas (TCGA) was analyzed using tools on the websites of The Human Protein Atlas (https://www.proteinatlas.org/) and Gene Expression Profiling Interactive Analysis (GEPIA; http://gepia.cancer-pku.cn/). Then, CRC-related gene expression and clinical data were downloaded from the cBioPortal (http://www.cbioportal.org) and the University of California Santa Cruz Xena (https://xena.ucsc.edu/) databases, while annotated gene sets were downloaded from the Kyoto Encyclopedia of Genes and Genomes (KEGG; http://www.genome.jp/kegg/pathway.html) and Gene Ontology (GO; http://www.geneontolog y.org/) databases. Data were enriched and analyzed using gene set enrichment analysis (GSEA) software to investigate the signaling pathways and biological processes potentially regulated by YTHDC2 in CRC.

\section{Immunofluorescence analysis}

Immunofluorescence was used to evaluate the localization of YTHDC2 in Caco2 cells. A single-cell suspension of $\mathrm{Caco} 2$ cells was aliquoted into 24 -well plates. When the cell density reached $85 \%-100 \%$, cells were fixed with $4 \%$ paraformaldehyde for $20 \mathrm{~min}$ at room temperature then drilled with $0.3 \%$ TritonX100 for $1 \mathrm{~h}$. After blocking with 5\% bovine serum albumin for 30 min, cells were incubated with Anti- 
YTHDC2 for $1 \mathrm{~h}$ and then with FITC- and TRITC-labeled secondary antibodies in dark conditions for 0.5-1 h. Finally, cells were counterstained with 4', 6-diamidino-2-phenylindole (DAPI, Southern Biotechnology Associates, USA) and observed under a confocal microscope (Nikon, Japan).

\section{Flow cytometry}

Transfected HCT116 and Caco2 cells were resuspended and $5 \times 10^{5}$ cells were counted and collected by flow cytometry. Binding buffer $(500 \mu \mathrm{l})$, Annexin V-FITC reagent $(5 \mu \mathrm{l})$ and propidium iodide reagent $(5 \mu \mathrm{l})$ were added successively, and the mixture incubated in dark conditions for 5-15 min at room temperature. The excitation wavelength $(E x)=488 \mathrm{~nm}$ and emission wavelength $(E m)=530 \mathrm{~nm}$. Cells were detected within $1 \mathrm{~h}$.

\section{Wound healing}

At 24h post-transfection, a straight scratch was made in the center of each well using a micropipette tip. Free suspended cells were washed with aseptic phosphate-buffered saline, and the solution imaged using a microscope at $0,12,24$, and $48 \mathrm{~h}$ after scratching.

\section{Reverse transcriptase-polymerase chain reaction}

After transfection, total RNA was isolated using the TRIzol Reagent Kit (Invitrogen) was used to isolate total RNA, and cDNA was reverse transcribed using the Quantscript RT kit (Tiangen, China). Transcript quantification was then performed using the SYBR RT-PCR kit (Tiangen) and specific RNA primers. Details of the RNA primers (SBS Genetech Co., Beijing, China) are provided in Table 1. The thermocycler program was: $95^{\circ} \mathrm{C}$ for $15 \mathrm{~min}$, then 40 cycles of $95^{\circ} \mathrm{C}$ for $10 \mathrm{~s}$, and an annealing step of $60^{\circ} \mathrm{C}$ for $32 \mathrm{~s}$. Expression levels of target genes were normalized using the $2^{-\triangle \Delta C t}$ method with GAPDH as an internal control.

\section{Western blot}

Transfected HCT116 and Caco2 cells were harvested and lysed using radioimmunoprecipitation assay (RIPA) lysis buffer (KeyGEN BioTECH, Jiangsu, China). After measuring the total protein concentration, the protein samples were separate by sodium dodecyl sulfate polyacrylamide gel electrophoresis (SDSPAGE), and then, electrotransferred to polyvinylidene fluoride membranes (PVDF). The PVDF membranes were blocked with $5 \%$ fat-free milk, and incubated with the primary antibody at $4{ }^{\circ} \mathrm{C}$ overnight, then with the secondary antibody for $1 \mathrm{~h}$ at room temperature. Finally, the Chemi Doc XRS System (Bio-Rad, Hercules, CA, USA) was used to analyze the protein bands with GAPDH as an internal control.

\section{Statistical analysis}

We used the SPSS 23.0 software (IBM Corp., Armonk, NY, USA) for statistical analysis. An independent samples $t$-test was used to compare two groups, while one-way analysis of variance (ANOVA) was used 
to compare three or more groups, and the least-square difference method was used for pairwise comparison after ANOVA. We considered $P<0.05$ as statistically significant.

\section{Results}

\section{Bioinformatics analysis}

Survival analysis of TCGA samples using The Human Protein Atlas showed high expression of YTHDC2 to be associated with higher overall survival in patients with colon cancer $\left(P=1.0 \times 10^{-3}\right)$, rectal cancer $(P$ $\left.=1.5 \times 10^{-5}\right)$, or both $\left(P=1.8 \times 10^{-5}\right)$ (Figure 1A). The results of GEPIA analysis showed the expression of YTHDC2 in tumor tissues of patients with CRC to be decreased compared with normal tissues $(P>0.05$, Figure 1B) and positively correlated with tumor stage ( $P=0.06$, Figure 1C); however, the difference was not statistically significant.

The results of KEGG enrichment analysis showed the expression of YTHDC2 in CRC to be related to the mitogen-activated protein kinase (MAPK) signaling pathway (effect size $[E S]=0.44$, false discovery rate $[\mathrm{FDR}]=0.118, P=0.028$ ), while GO enrichment analysis indicated YTHDC2 to be related to the intrinsic apoptotic signaling pathway $(E S=0.47, F D R=0.053, P=0.013)$, regulation of extrinsic apoptotic signaling pathway $(E S=0.45, F D R=0.069, P=0.019)$, and cell cycle arrest $(E S=0.46, F D R=0.057, P=$ 0.01 ) (Figure 1D). Since the p38MAPK signaling pathway is mainly related to apoptosis, YTHDC2 may promote apoptosis of CRC cells via regulation of the p38MAPK signaling pathway.

Analysis the correlation between YTHDC2 and various apoptotic proteins downstream of the MAPK signaling pathway using the GEPIA website revealed that, in the exogenous death receptor activation pathway, representative apoptotic proteins Caspase $8(P=0, \mathrm{R}=0.53)$, Fas $(P=0, \mathrm{R}=0.44)$ and Fas $\mathrm{L}(P$ $=3.2 \times 10^{-6}, R=0.24$ ) were significantly positively correlated with YTHDC2. In the endogenous mitochondrial apoptosis-related pathway, representative apoptosis proteins including $\operatorname{Bax}\left(P=3.5 \times 10^{-6}, \mathrm{R}\right.$ $=0.24)$, Caspase $9\left(P=2.2 \times 10^{-6}, \mathrm{R}=0.24\right)$ and Cytochrome $\mathrm{C}\left(P=3.9 \times 10^{-5}, \mathrm{R}=0.21\right)$ were found to be significantly positively correlated with YTHDC2, although the correlation coefficient was relatively small. However, in the endoplasmic reticulum apoptosis pathway, the correlation coefficient of the apoptotic protein Caspase 12 was small $\left(P=1.9 \times 10^{-2}, \mathrm{R}=0.12\right)$. Therefore, YTHDC2-mediated apoptosis may be mainly related to the exogenous death receptor activation pathway and the endogenous mitochondrial apoptosis-related pathway.

\section{Localization of YTHDC2 in Caco2 cells}

The Caco2 cell line exhibited high expression of YTHDC2 and was used for immunofluorescence experiments, which showed YTHDC2 to be mainly located in the nucleus and perinuclear region (Figure 2A).

\section{Significantly promotion of apoptosis was induced by YTHDC2}


In HCT116 cells, the apoptosis rate of the YTHDC2 group was significantly higher (18.44\%) than that of the con-YTHDC2 (11.94\%) and siYTHDC2 (7.13\%) groups. The apoptosis rate of the siYTHDC2 group was decreased compared with that of the NC-siYTHDC2 (9.46\%) group. Similar results were observed for Caco2 2 cells; the apoptosis rate in the con-YTHDC2, YTHDC2, NC-siYTHDC2, and siYTHDC2 groups were $11.96 \%, 16.40 \%, 10.73 \%$, and $9.62 \%$, respectively (Figure 2B). These results indicate that YTHDC2 promotes apoptosis of CRC cells.

\section{Metastasis and invasion ability of HCT116 and Caco2 cells is reduced by YTHDC2}

Wound healing analysis revealed that the growth of HCT116 cells was significantly different between all transfection groups $(P=0.000)$. After $48 \mathrm{~h}$, the growth velocity of the YTHDC2 group was significantly lower than that of the con-YTHDC2 and NC-siYTHDC2 groups $(P=0.003)$, while the growth rate of the siYTHDC2 group was significantly higher than that of the NC-siYTHDC2 group $(P=0.000)$. The difference between the YTHDC2 and siYTHDC2 groups was highly significant $(P=0.000)$. In Caco2 cells, the differences between each group were also significant $(P=0.000)$ (Figure 2C). These results showed that YTHDC2 inhibited the metastasis and invasion of tumor cells and promoted the prognosis of CRC, which was consistent with the results of bioinformatics analysis.

\section{Overexpression of YTHDC2 promotes activation of p-p38MAPK}

Western blot analysis revealed that the relative expression level of p38MAPK was not significantly different among the four groups in either HCT116 or Caco2 cells (HCT116: $P=0.775$; Caco2: $P=0.851$ ); while p-p38MAPK levels were significantly different (HCT116: $P=0.002$; Caco2: $P=0.037$ ); the YTHDC2 group showed significantly higher expression than the con-YTHDC2 (HCT116: $P=0.046$, Caco2: $P=$ $0.035)$ or siYTHDC2 groups (HCT116: $P=0.000$, Caco2: $P=0.007)$. The $p-p 38 M A P K$ level of the siYTHDC2 group was significantly lower than the that of NC-siYTHDC2 group in HCT116 cells $(P=$ 0.002). In Caco 2 cells, consistent with HCT116 cells, the siYTHDC2 group showed decreased expression compared with the NC-siYTHDC2 group, although this was not statistically significant $(P=0.106)$, (Figure 3). These results indicate that, although YTHDC2 does not promote the basic expression of p38MAPK, it significantly increases the activation of p38MAPK, causing overactivation of the p38MAPK signaling pathway and promoting of tumor cell apoptosis.

\section{Up-regulation of apoptosis-related proteins downstream of the MAPK signaling pathway is induced by YTHDC2.}

In the exogenous death receptor activation pathway, the mRNA and protein expression of Caspase 8 was not statistically different in HCT116 cells $(P=0.667 ; P=0.229)$, while this difference was significant in Caco 2 cells $(P=0.000 ; P=0.000)$. The results of RT-PCR and Western blot revealed significant differences in the mRNA and protein expression of apoptin Fas in both HCT116 $(P=0.000 ; P=0.000)$ and Caco2 cells $(P=0.000 ; P=0.001)$. For Fas $L$, the mRNA expression was significantly increased in the YTHDC2 group and significantly decreased in the siYTHDC2 group in both HCT116 and Caco2 cells $(P=$ $0.002 ; P=0.000)$. In addition, Western blot showed the expression of Fas $L$ to be unchanged with 
changes in YTHDC2 expression, and no significant differences were detected among the four groups in HCT 116 cells $(P=0.874)$. In Caco2 cells, the expression of Fas $L$ protein was not significantly different to that of YTHDC2 or Caspase $8(P=0.010)$. The difference in expression levels of Caspase 8 and Fas $L$ in HCT116 and Caco2 cells may be due to different expression levels of YTHDC2 between these two cell lines (Figure 4A, 4B).

In the endogenous mitochondrial apoptosis-related pathway, the mRNA and protein expression of Bax in HCT116 cells was significantly different in each group $(P=0.000 ; P=0.004)$. This was also observed for Caco 2 cells $(P=0.000 ; P=0.001)$. The mRNA expression of Caspase 9 was not statistically different between the siYTHDC2 and NC-siYTHDC2 groups in either cell line $(P=0.446 ; P=0.129)$, but a significant difference was observed among the other groups (all $P<0.05$ ). The protein expression of Caspase 9 differed among the groups in both HCT116 and Caco 2 cell lines ( $P=0.008 ; P=0.007)$. In HCT116 cells, there was no significant difference in Cytochrome $C$ mRNA expression among any groups (all $P>0.05$ ), except for the YTHDC2 and siYTHDC2 groups $(P=0.011)$. In Caco2 cells, the mRNA expression of Cytochrome $C$ was similar to that of Caspase $9(P=0.000)$. Furthermore, the protein expression of Cytochrome $C$ was significantly affected by changes in YTHDC2 expression in HCT116 and Caco2 cells $(P=0.000 ; P=0.003)$. Although not all apoptotic proteins showed statistically significant differences, the variations in expression were found to reflect changes in YTHDC2 expression, indicating that changes in YTHDC2 affect the endogenous mitochondrial apoptosis-related pathway (Figure 4C, 4D).

In the endoplasmic reticulum apoptosis pathway, RT-PCR (HCT116: $P=0.519$; Caco2: $P=0.665$ ) and Western blot (HCT116: $P=0.213$; Caco2: $P=0.847$ ) revealed that the expression of apoptin Caspase 12 was not significantly different among the four groups in HCT116 or in Caco2 cells. Therefore, the expression of Caspase 12 does not change in line with changes in YTHDC2 expression, indicating that YTHDC2-mediated promotion of apoptosis of CRC cells is not effected via the endogenous endoplasmic reticulum apoptosis pathway (Figure 4E, 4F).

\section{Discussion}

As an $\mathrm{m}^{6} \mathrm{~A}$-binding protein, YTHDC2 is involved in a variety of biological processes. Many studies have indicated YTHDC2 to be crucial for fertility in both male and female mice, with particular importance in spermatogenesis ${ }^{[21-24]}$. Other studies have indicated that YTHDC2 may be related to autism spectrum disorder ${ }^{[25]}$, hepatitis $\mathrm{C}^{[21]}$, pancreatic adenocarcinoma ${ }^{[26]}$, and $\mathrm{CRC}^{[27]}$. The present research demonstrates that the expression of YTHDC2 is negatively correlated with overall survival and tumor stage in CRC. Overexpression of YTHDC2 causes the apoptosis rate to increase in HCT116 and Caco2 cells, and to reduce the growth rate of CRC cells. These results indicate that YTHDC2 can inhibits the metastasis and invasion of tumor cells, improving the prognosis of CRC and acting as a protective factor for this disease. However, this is in contrast to the results of Tanabe et al. ${ }^{[27]}$, who suggest that YTHDC2 may contribute to colon cancer metastasis by promoting the translation of hypoxia-inducible factor-1 alpha (HIF-1a), the expression of which is positively correlated with tumor stage. This discrepancy may be due to the different targets of YTHDC2 compared with HIF-1】. 
The MAPKs are Ser/Thr protein kinases which are found in a wide variety of cell types. Multiple parallel MAPK signaling pathways have been identified in mammalian cells, including the extracellular-signalregulated kinase $1 / 2\left(\right.$ ERK1/2) ${ }^{[27]}$, c-Jun N-terminal kinase/stress-activated protein kinase (JNK/SAPK) ${ }^{[29]}$, p38MAPK ${ }^{[30]}$, and extracellular-signal-regulated kinase 5/Big MAP kinase I (ERK5/BMKI) ${ }^{[31]}$ pathways. Activation of p38MAPK-a key component of the MAPK signaling pathway-depends on phosphorylation of Tyr and Thr sites; phosphorylated p38MAPK plays an important role in the regulation of cell proliferation, differentiation, migration, apoptosis, and death. Studies have shown that targeted inhibition of p38MAPK activity can alleviate rheumatoid arthritis ${ }^{[32]}$, asthma ${ }^{[33]}$, and other inflammationassociated diseases. Other studies have indicated that excessive activation of p38MAPK can significantly promote apoptosis of hepatocellular carcinoma cells, thus improving the prognosis of hepatocellular carcinoma ${ }^{[34]}$. The p38MAPK signaling pathway regulates apoptosis via Fas/Fas Lmediated apoptosis, induction of the translocation of Bax, and enhancement of C-myc expression and p53 phosphorylation, among other mechanisms. Our results of GSEA enrichment analysis demonstrate that YTHDC2 is significantly correlated with the MAPK signaling pathway in CRC and regulates biological processes such as the intrinsic apoptotic signaling pathway, regulation of extrinsic apoptotic signaling pathway, and cell cycle arrest. On the one hand, p38MAPK was found to be closely related to apoptosis in the MAPK signaling pathway, where it initiates cell apoptosis and is related to cell cycle quiescence. On the other hand, cytological research revealed that up- or downregulation of YTHDC2 and phosphorylation of p38MAPK change with altered YTHDC2 expression. Flow cytometry and wound healing assays also showed that YTHDC2 promotes apoptosis of CRC cells. Taken together, these findings indicate that YTHDC2 promotes apoptosis of CRC cells through the p38MAPK signaling pathway.

There are three kinds of apoptotic signaling pathways: the exogenous death receptor activation pathway, the endogenous mitochondrial apoptosis-related pathway, and the endoplasmic reticulum apoptosis pathway, each with their own specific apoptotic proteins. In the exogenous death receptor activation pathway these are Fas, Fas L, and Caspase 8; in the endogenous mitochondrial apoptosis-related pathway, Bax, Caspase 9, and Cytochrome C; in the endoplasmic reticulum apoptosis pathway, Caspase 12. We found expression of the above apoptosis-related proteins to be positively correlated with that of YTHDC2; the correlation coefficients of Fas, Fas L, and Caspase 8 were generally large while those of Bax, Caspase 9, and Cytochrome $C$ were relatively small. The correlation coefficient of Caspase 12 was found to be the smallest. These findings were reflected by the results of Western blot and RT-PCR, suggesting that YTHDC2 promotes apoptosis of CRC cells through activation of the exogenous death receptor and endogenous mitochondrial apoptosis-related pathways, rather than the endoplasmic reticulum apoptosis pathway.

\section{Conclusion}

In conclusion, overexpression of YTHDC2 can promote apoptosis of CRC cells and improve the prognosis of patients with CRC by regulating the p38MAPK signaling pathway. The apoptosis is effected by activation of the exogenous death receptor and endogenous mitochondrial apoptosis-related pathways. 
Further research into the mechanisms underlying YTHDC2-mediated apoptosis may inform the development of new strategies for the treatment of CRC.

\section{Abbreviations}

YTHDC2: YTH-domain containing. p38MAPK: p38 mitogen-activated protein kinase. CRC: colorectal cancer. TCGA: The Cancer Genome Atlas. $\mathrm{m}^{6} \mathrm{~A}: \mathrm{N}^{6}$-methyladenosin. METTL3: methyltransferase-like 3 . METTL14: methyltransferase-like 14. WTAP: Methyltransferase complex composition. ALKBH5: demethylases. FTO: demethylasesYTHDF: YTH-domain protein family. CCR4-NOT: carbon catabolite repressor 4-negative. HNRNP: heterogeneous nuclear ribonucleoprotein family. Anti-GAPDH: AntiGlyceraldehyde 3-phosphate dehydrogenase. CST: Cell Signaling Technology.FITC: Fluorescein isothiocyanate. TRITC: tetramethylrhodamine. ATCC: American Type Culture Collection. TCGA: The Cancer Genome Atlas. KEGG: Kyoto Encyclopedia of Genes and Genomes. GO: Ontology. GEPIA: Gene Expression Profiling Interactive Analysis GSEA: gene set enrichment analysis. DAPI: 6-diamidino-2phenylindole. RIPA: radioimmunoprecipitation assay. SDS-PAGE: sodium dodecyl sulfate polyacrylamide gel electrophoresis. PVDF: polyvinylidene fluoride membranes. ANOVA: analysis of variance. FDR: false discovery rate. JNK/SAPK: C-Jun N-terminal kinase/stress-activated protein kinase. ERK5/BMKI: extracellular-signal-regulated kinase 5/Big MAP kinase I. HRP: Horseradish peroxidase.

\section{Declarations}

Acknowledgments:We thank Amy Phillips, PhD, from Liwen Bianji, Edanz Editing China (www.liwenbianji.cn/ac), for editing the English text of a draft of this manuscript.

Funding Sources: No funding was received

Availability of data and materials: The datasets used and/ or analyzed during the current study are available from the corresponding author on reasonable request.

\section{Authors' contributions:}

Tao Yu: Writing- Original draft preparation.

Yanyan Xu: Writing- Reviewing and Editing

Tao Sun: Visualization, Investigation

Di Huang: Methodology, Supervision

Feng Cao: Data curation

Xin Gao: Software

Gang Liu: Conceptualization, Methodology, Software 
Ethics approval and consent to participate: The study was approved by the Ethics Committee of Medical Ethics Committee of the Tianjin Medical University General Hospital.

Patient consent for publication: No patients involved.

Competing interests: No conflict of interest was declared.

\section{References}

[1] Bray F, Ferlay J, Soerjomataram I, et al. Global cancer statistics 2018: GLOBOCAN estimates of incidence and mortality worldwide for 36 cancers in 185 countries[J]. CA Cancer J Clin. 2018, 68(6): 394424.

[2] Arnold M, Sierra M S, Laversanne M, et al. Global patterns and trends in colorectal cancer incidence and mortality[J]. Gut. 2017, 66(4): 683-691.

[3] Batista P J. The RNA Modification N(6)-methyladenosine and Its Implications in Human Disease[J]. Genomics Proteomics Bioinformatics. 2017, 15(3): 154-163.

[4] Hsu P J, Zhu Y, Ma H, et al. Ythdc2 is an N(6)-methyladenosine binding protein that regulates mammalian spermatogenesis[J]. Cell Res. 2017, 27(9): 1115-1127.

[5] Bokar J A, Shambaugh M E, Polayes D, et al. Purification and cDNA cloning of the AdoMet-binding subunit of the human mRNA (N6-adenosine)-methyltransferase[J]. RNA. 1997, 3(11): 1233-1247.

[6] Liu J, Yue Y, Han D, et al. A METTL3-METTL14 complex mediates mammalian nuclear RNA N6adenosine methylation[J]. Nat Chem Biol. 2014, 10(2): 93-95.

[7] Ping X L, Sun B F, Wang L, et al. Mammalian WTAP is a regulatory subunit of the RNA N6methyladenosine methyltransferase[J]. Cell Res. 2014, 24(2): 177-189.

[8] Zheng G, Dahl J A, Niu Y, et al. ALKBH5 is a mammalian RNA demethylase that impacts RNA metabolism and mouse fertility[J]. Mol Cell. 2013, 49(1): 18-29.

[9] Jia G, Fu Y, Zhao X, et al. N6-methyladenosine in nuclear RNA is a major substrate of the obesityassociated FTO[J]. Nat Chem Biol. 2011, 7(12): 885-887.

[10] Wang X, Lu Z, Gomez A, et al. N6-methyladenosine-dependent regulation of messenger RNA stability[J]. Nature. 2014, 505(7481): 117-120.

[11] Liu N, Dai Q, Zheng G, et al. N(6)-methyladenosine-dependent RNA structural switches regulate RNAprotein interactions[J]. Nature. 2015, 518(7540): 560-564.

[12] Xu C, Wang X, Liu K, et al. Structural basis for selective binding of m6A RNA by the YTHDC1 YTH domain[J]. Nat Chem Biol. 2014, 10(11): 927-929. 
[13] Shi H, Wang X, Lu Z, et al. YTHDF3 facilitates translation and decay of N(6)-methyladenosinemodified RNA[J]. Cell Res. 2017, 27(3): 315-328.

[14] Du H, Zhao Y, He J, et al. YTHDF2 destabilizes m(6)A-containing RNA through direct recruitment of the CCR4-NOT deadenylase complex[J]. Nat Commun. 2016, 7: 12626.

[15] Xiao W, Adhikari S, Dahal U, et al. Nuclear m(6)A Reader YTHDC1 Regulates mRNA Splicing[J]. Mol Cell. 2016, 61(4): 507-519.

[16] Kretschmer J, Rao H, Hackert P, et al. The m(6)A reader protein YTHDC2 interacts with the small ribosomal subunit and the 5'-3' exoribonuclease XRN1[J]. RNA. 2018, 24(10): 1339-1350.

[17] Zhao B S, Wang X, Beadell A V, et al. m(6)A-dependent maternal mRNA clearance facilitates zebrafish maternal-to-zygotic transition[J]. Nature. 2017, 542(7642): 475-478.

[18] Geula S, Moshitch-Moshkovitz S, Dominissini D, et al. Stem cells. m6A mRNA methylation facilitates resolution of naive pluripotency toward differentiation[J]. Science. 2015, 347(6225): 1002-1006.

[19] Fanale D, lovanna J L, Calvo E L, et al. Germline copy number variation in the YTHDC2 gene: does it have a role in finding a novel potential molecular target involved in pancreatic adenocarcinoma susceptibility?[J]. Expert Opin Ther Targets. 2014, 18(8): 841-850.

[20] Tanabe A, Konno J, Tanikawa K, et al. Transcriptional machinery of TNF-alpha-inducible YTH domain containing 2 (YTHDC2) gene[J]. Gene. 2014, 535(1): 24-32.

[21] Jain D, Puno M R, Meydan C, et al. ketu mutant mice uncover an essential meiotic function for the ancient RNA helicase YTHDC2[J]. Elife. 2018, 7.

[22] Soh Y, Mikedis M M, Kojima M, et al. Meioc maintains an extended meiotic prophase I in mice[J]. PLoS Genet. 2017, 13(4): e1006704.

[23] Wojtas M N, Pandey R R, Mendel M, et al. Regulation of m(6)A Transcripts by the 3'--\&gt;5' RNA Helicase YTHDC2 Is Essential for a Successful Meiotic Program in the Mammalian Germline[J]. Mol Cell. 2017, 68(2): 374-387.

[24] Bailey A S, Batista P J, Gold R S, et al. The conserved RNA helicase YTHDC2 regulates the transition from proliferation to differentiation in the germline[J]. Elife. 2017, 6.

[25] Liu X, Shimada T, Otowa T, et al. Genome-wide Association Study of Autism Spectrum Disorder in the East Asian Populations[J]. Autism Res. 2016, 9(3): 340-349.

[26] Fanale D, lovanna J L, Calvo E L, et al. Germline copy number variation in the YTHDC2 gene: does it have a role in finding a novel potential molecular target involved in pancreatic adenocarcinoma susceptibility?[J]. Expert Opin Ther Targets. 2014, 18(8): 841-850. 
[27] Tanabe A, Tanikawa K, Tsunetomi M, et al. RNA helicase YTHDC2 promotes cancer metastasis via the enhancement of the efficiency by which HIF-1alpha mRNA is translated[J]. Cancer Lett. 2016, 376(1): $34-42$.

[28] Sturgill T W, Ray L B. Muscle proteins related to microtubule associated protein-2 are substrates for an insulin-stimulatable kinase[J]. Biochem Biophys Res Commun. 1986, 134(2): 565-571.

[29] Barr R K, Bogoyevitch M A. The c-Jun N-terminal protein kinase family of mitogen-activated protein kinases (JNK MAPKs) [J]. Int J Biochem Cell Biol. 2001, 33(11): 1047-1063.

[30] Zou X, Blank M. Targeting p38 MAP kinase signaling in cancer through post-translational modifications[J]. Cancer Lett. 2017, 384: 19-26.

[31] Zhou G, Bao Z Q, Dixon J E. Components of a new human protein kinase signal transduction pathway[J]. J Biol Chem. 1995, 270(21): 12665-12669.

[32] Schett G, Zwerina J, Firestein G. The p38 mitogen-activated protein kinase (MAPK) pathway in rheumatoid arthritis[J]. Ann Rheum Dis. 2008, 67(7): 909-916.

[33] Adcock I M, Caramori G, Chung K F. New targets for drug development in asthma[J]. Lancet. 2008, 372(9643): 1073-1087.

[34] Wang Y, Xia C, Lv Y, et al. Crosstalk Influence between P38MAPK and Autophagy on MitochondriaMediated Apoptosis Induced by Anti-Fas Antibody/Actinomycin D in Human Hepatoma Bel-7402 Cells[J]. Molecules. 2017, 22(10).

\section{Tables}

Table 1 Primers used for RT-PCR 


\begin{tabular}{|c|c|c|}
\hline & Oligonucleotide sequence ( $\left.5^{\prime}-3^{\prime}\right)$ & Tm value $\left({ }^{\circ} \mathrm{C}\right)$ \\
\hline \multicolumn{3}{|l|}{ YTHDC2 } \\
\hline Forward & 5'-AGATAAGCAGGGATGGGCAG -3' & 59.23 \\
\hline Reverse & 5'-AAGACGTTCCCATAACTGGAGC -3' & 60.35 \\
\hline \multicolumn{3}{|l|}{ Casp9 } \\
\hline Forward & 5'-CACTGGCTCCAACATCGACT -3' & 60.04 \\
\hline Reverse & 5'-CACCGAAACAGCATTAGCGAC -3' & 60.20 \\
\hline \multicolumn{3}{|l|}{$\mathrm{Bcl}-2$} \\
\hline Forward & 5'-GTGAACTGGGGGAGGATTGT -3' & 59.30 \\
\hline Reverse & 5'-CCGTACAGTTCCACAAAGGCA -3' & 60.81 \\
\hline \multicolumn{3}{|l|}{ BAX } \\
\hline Forward & 5'-ACTGAAGCGACTGATGTCCC -3' & 59.75 \\
\hline Reverse & 5'-CTCCCGCCACAAAGATGGT -3' & 60.00 \\
\hline \multicolumn{3}{|l|}{ CYCS } \\
\hline Forward & 5'-ATTATGAAGTGTTCCCAGTGCC -3' & 58.64 \\
\hline Reverse & 5'-GCTGTGTAAGAGTATCCAGGGG -3' & 59.9 \\
\hline \multicolumn{3}{|l|}{ Casp 8} \\
\hline Forward & 5'- CTGCCTACAGGGTCATGCTC-3' & 60.18 \\
\hline Reverse & 5'-AСААСТССТССССТTТGCTG -3' & 59.89 \\
\hline \multicolumn{3}{|l|}{ FAS } \\
\hline Forward & 5'-GGACCCTCCTACCTCTGGTT -3' & 59.96 \\
\hline Reverse & 5'- GAGGACAGGGCTTATGGCAG -3' & 60.18 \\
\hline \multicolumn{3}{|l|}{ FASL } \\
\hline Forward & 5'-GGTTCTGGTTGCCTTGGTAGGA -3' & 62.21 \\
\hline Reverse & 5'-CTGTGTGCATCTGGCTGGTAGA -3' & 62.31 \\
\hline \multicolumn{3}{|l|}{ Casp12 } \\
\hline Forward & 5'-TTGACCTTTTGGGGATGCGA -3' & 59.89 \\
\hline Reverse & 5'-GCTTGGTCCCACAGATTCCA -3' & 59.96 \\
\hline \multicolumn{3}{|l|}{ GAPDH } \\
\hline Forward & 5'-GTCTCCTCTGACTTCAACAGCG-3' & 60.92 \\
\hline Reverse & 5'-ACCACCCTGTTGCTGTAGCCAA-3' & 64.41 \\
\hline
\end{tabular}

\section{Figures}



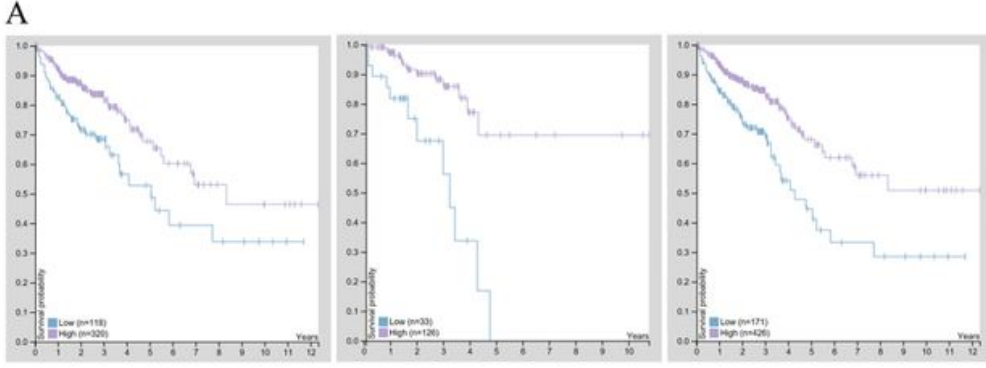

D
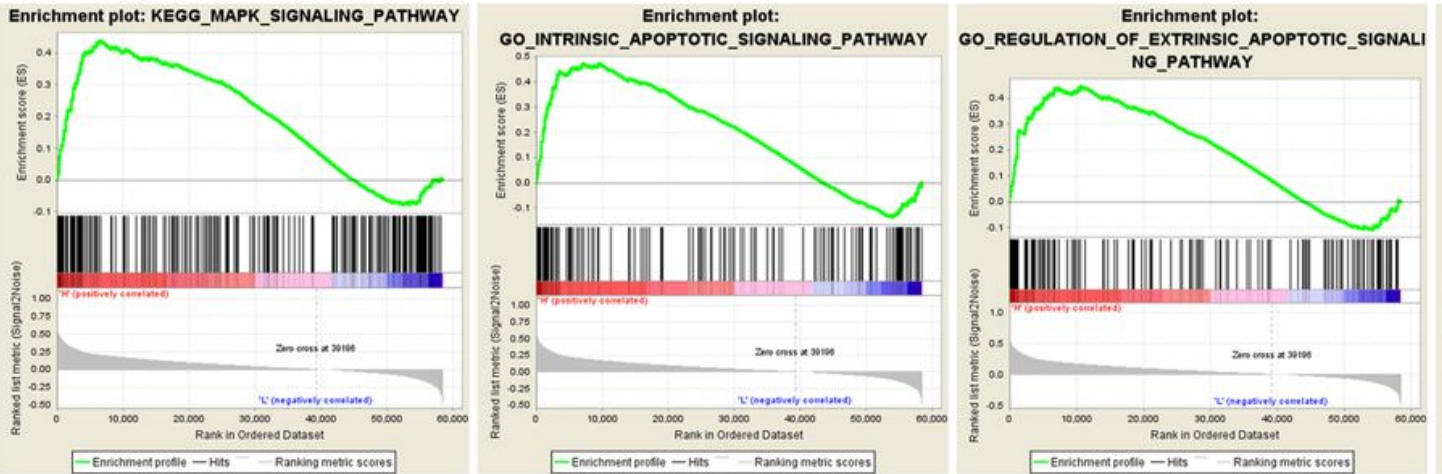

E

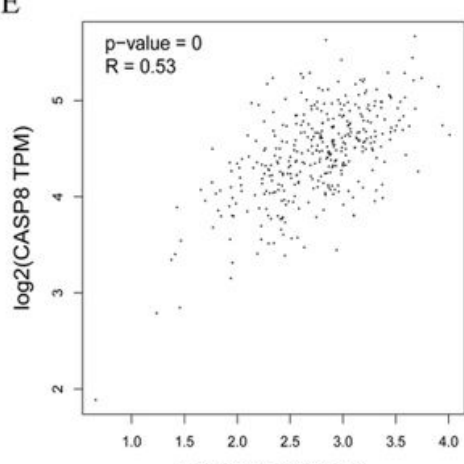

$\mathrm{F}$
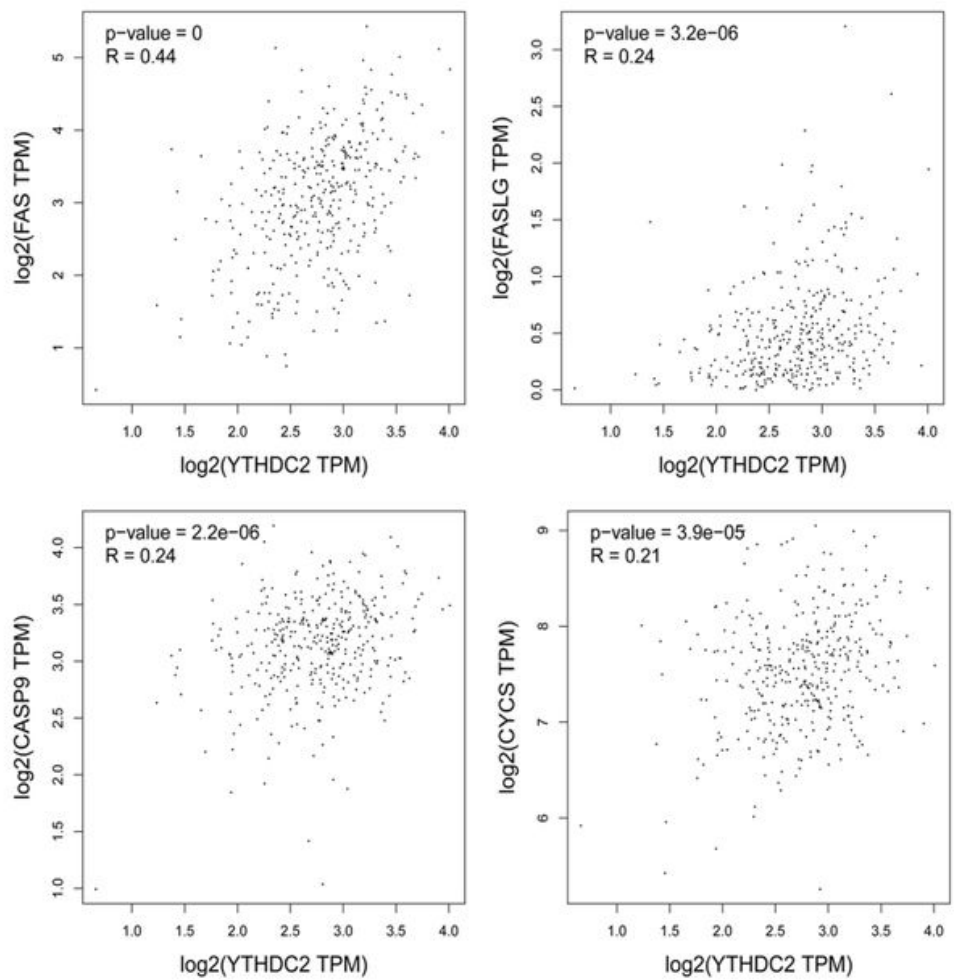

B
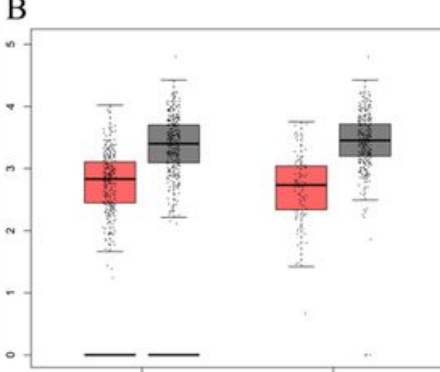

$\mathrm{C}$

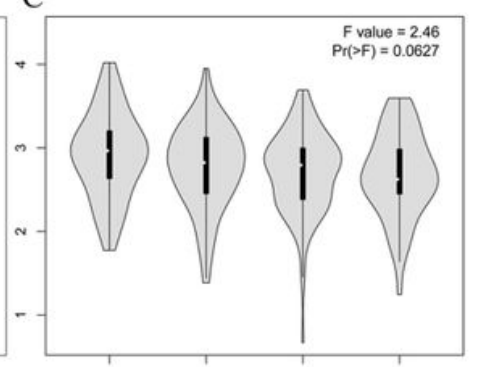

Stage 1 Stage II Stage III Stage IV

Enrichment plot: GO_CELL_CYCLE_ARREST
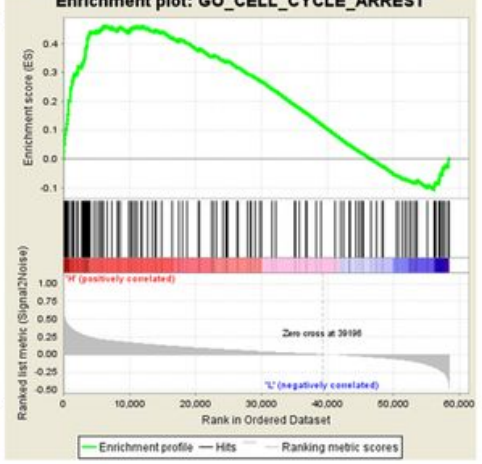

G

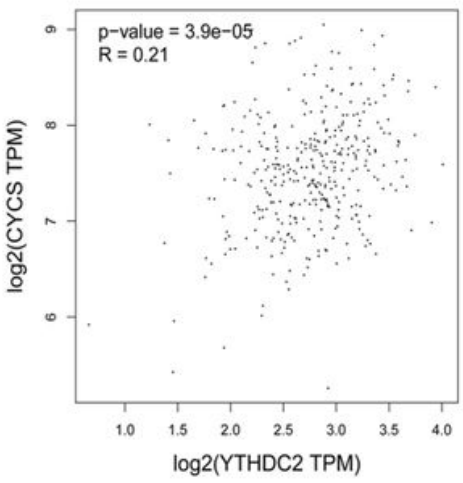

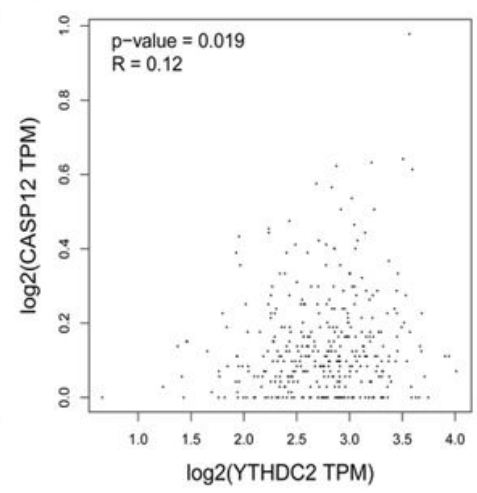

Figure 1

Bioinformatics analysis. A) Survival analysis of the YTH-domain family protein YTHDC2 in patients with colon cancer, rectal cancer, or colorectal cancer patients. B) Expression levels of YTHDC2 in tumor tissues of patients with colorectal cancer. C) The relationship between YTHDC2 and tumor stage. D) Gene set enrichment analysis. E) Correlation between the expression levels of YTHDC2 and apoptotic proteins of the exogenous death receptor activation pathway. F) Correlation between the expression of YTHDC2 and 
apoptotic proteins of the endogenous mitochondrial apoptosis-related pathway; G) Correlation between the expression of YTHDC2 and apoptotic proteins of the endoplasmic reticulum apoptosis pathway.
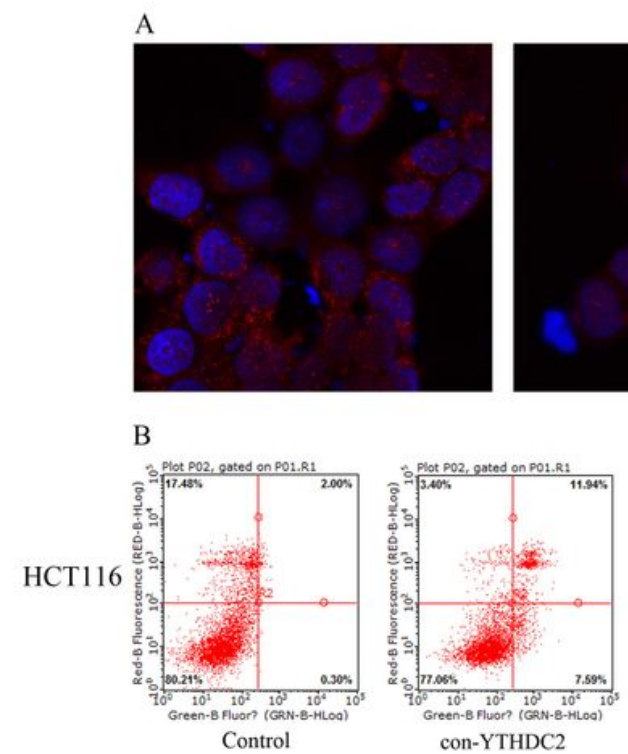

con-YTHDC2

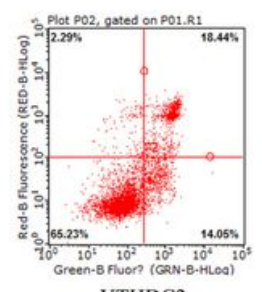

YTHDC2
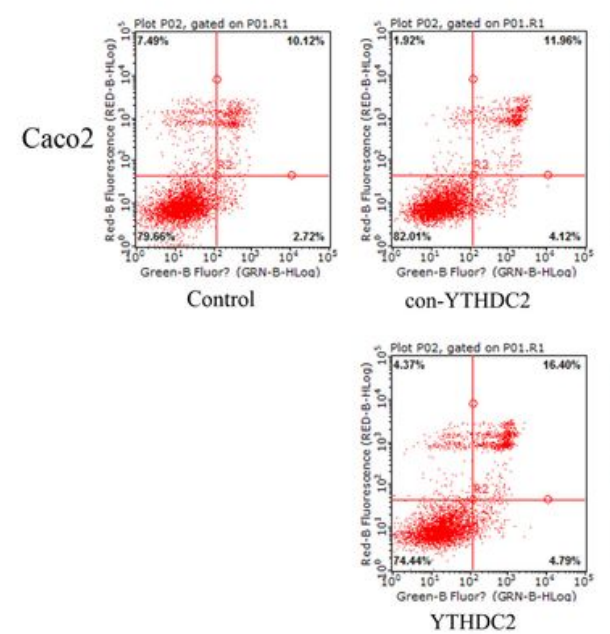

YTHDC2
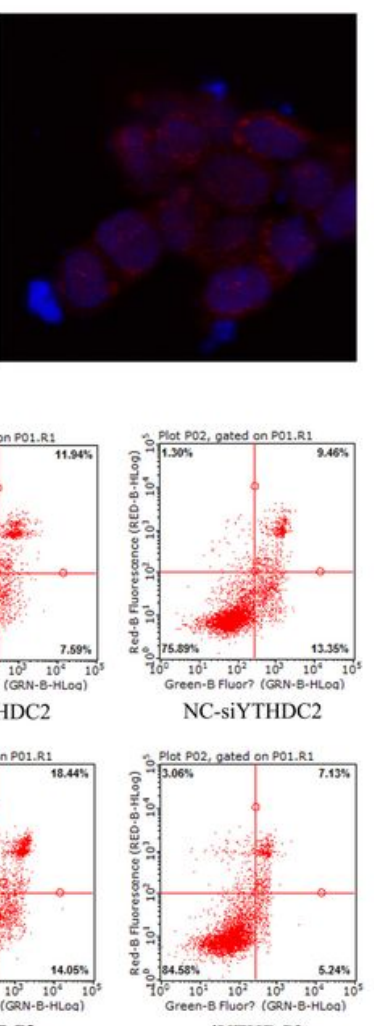

siYTHDC2
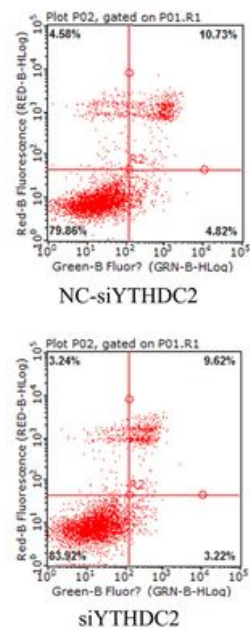
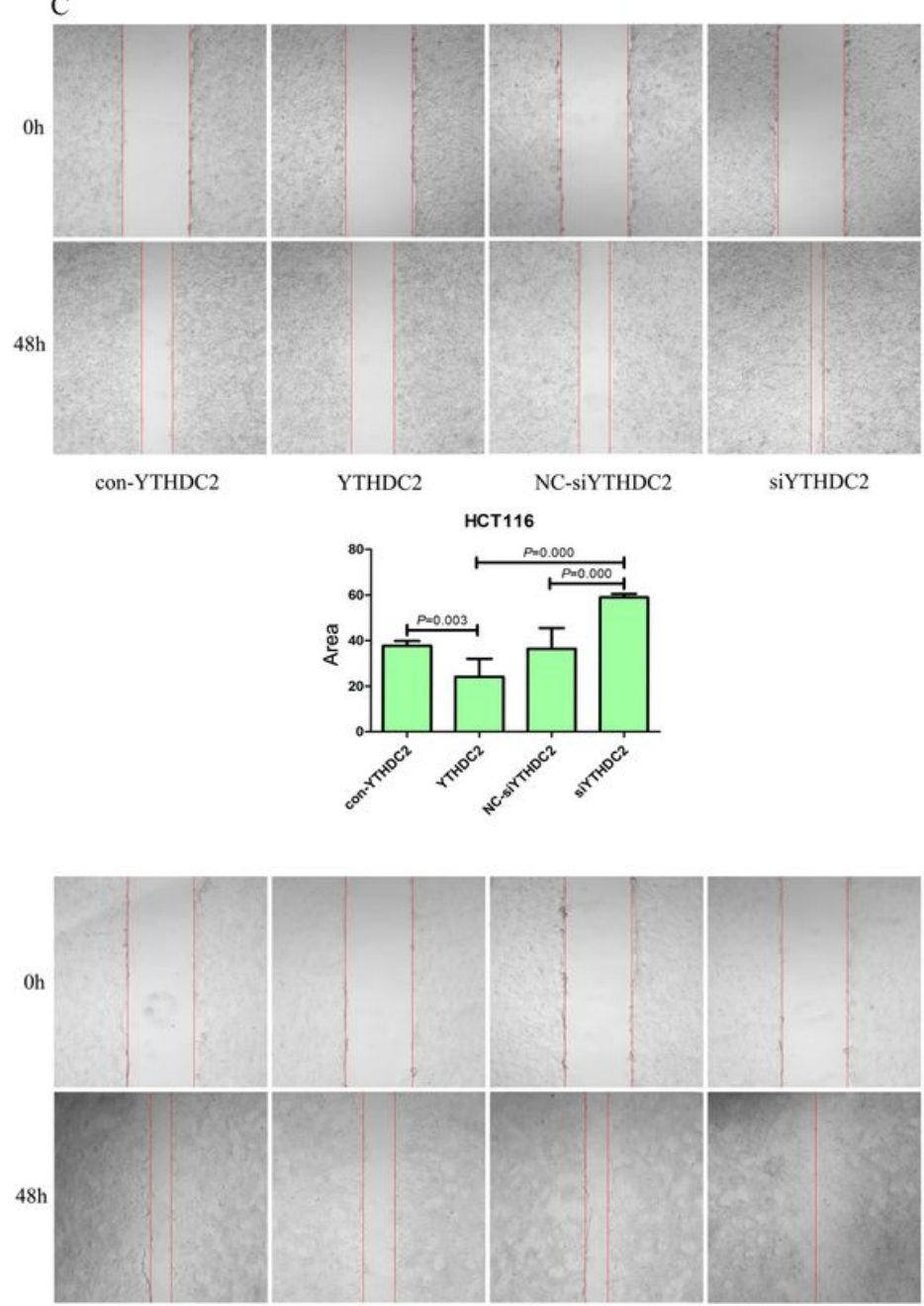

con-YTHDC2

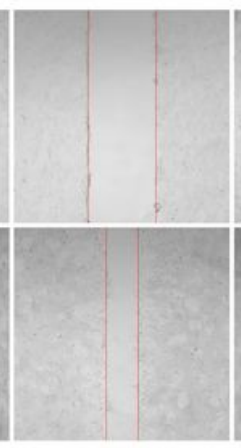

YTHDC2

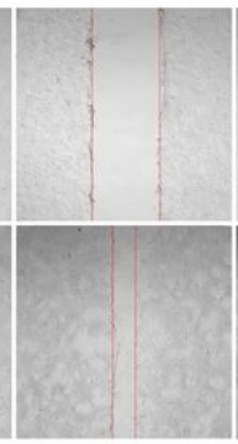

NC-siYTHDC2

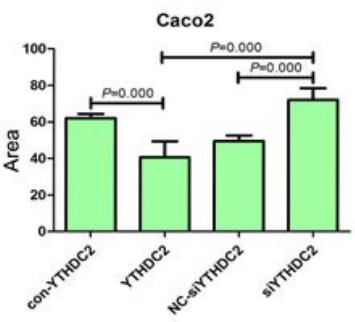

\section{Figure 2}

Changes in apoptosis, metastasis, and invasion of colorectal cancer cell lines. A) Representative microscopy images showing the localization of YTHDC2 in Caco2 cells. B) Changes in the level of apoptosis in relation to knockdown or overexpression of YTHDC2. C) Changes in the invasion and metastasis abilities in response to knockdown and overexpression YTHDC2. 

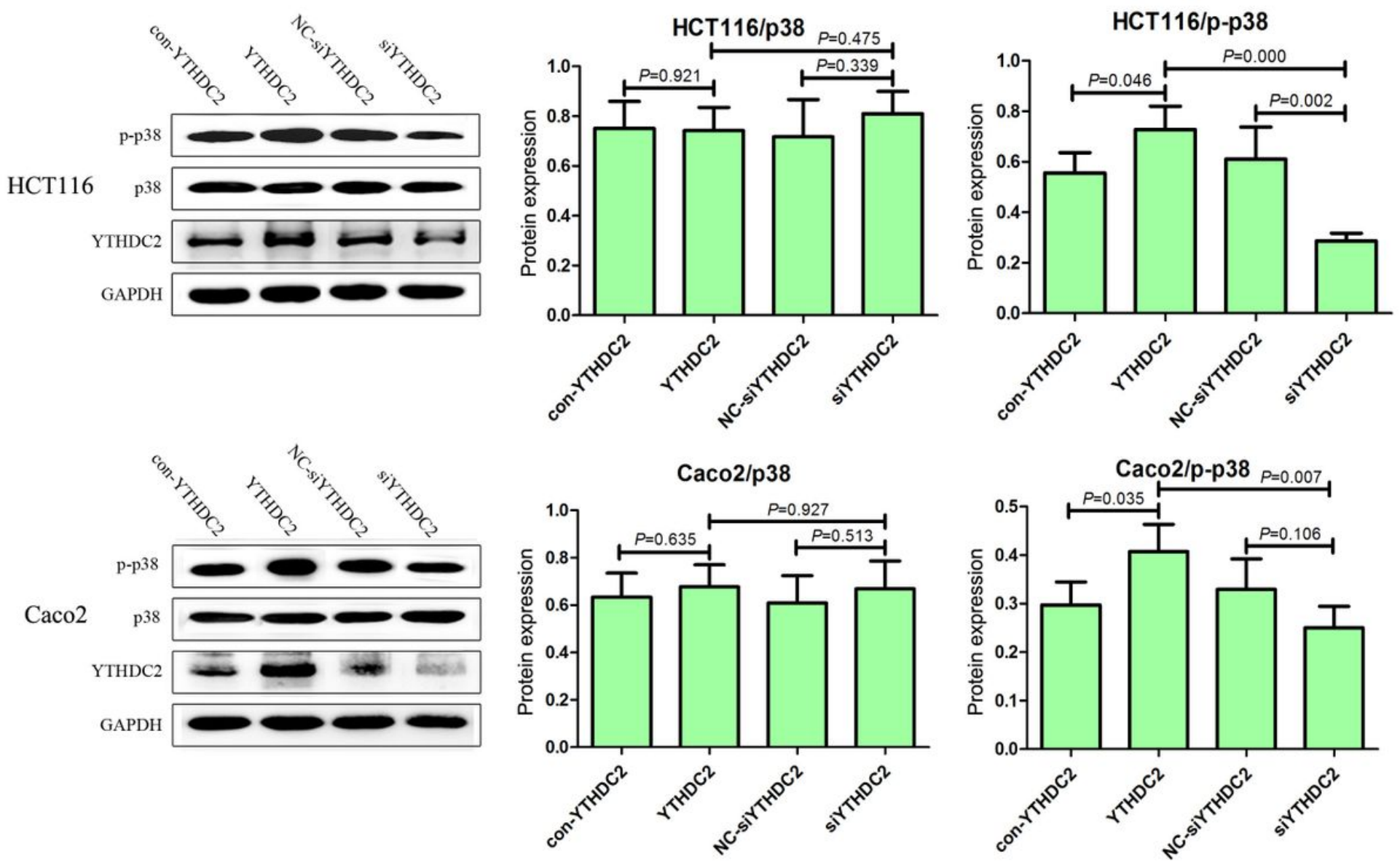

Figure 3

The influence of YTHDC2 on the p38 mitogen-associated protein kinase signal pathway: Levels of p38 mitogen-associated protein kinase (MAPK) and p-p38MAPK protein in response to knockdown or overexpression YTHDC2. 


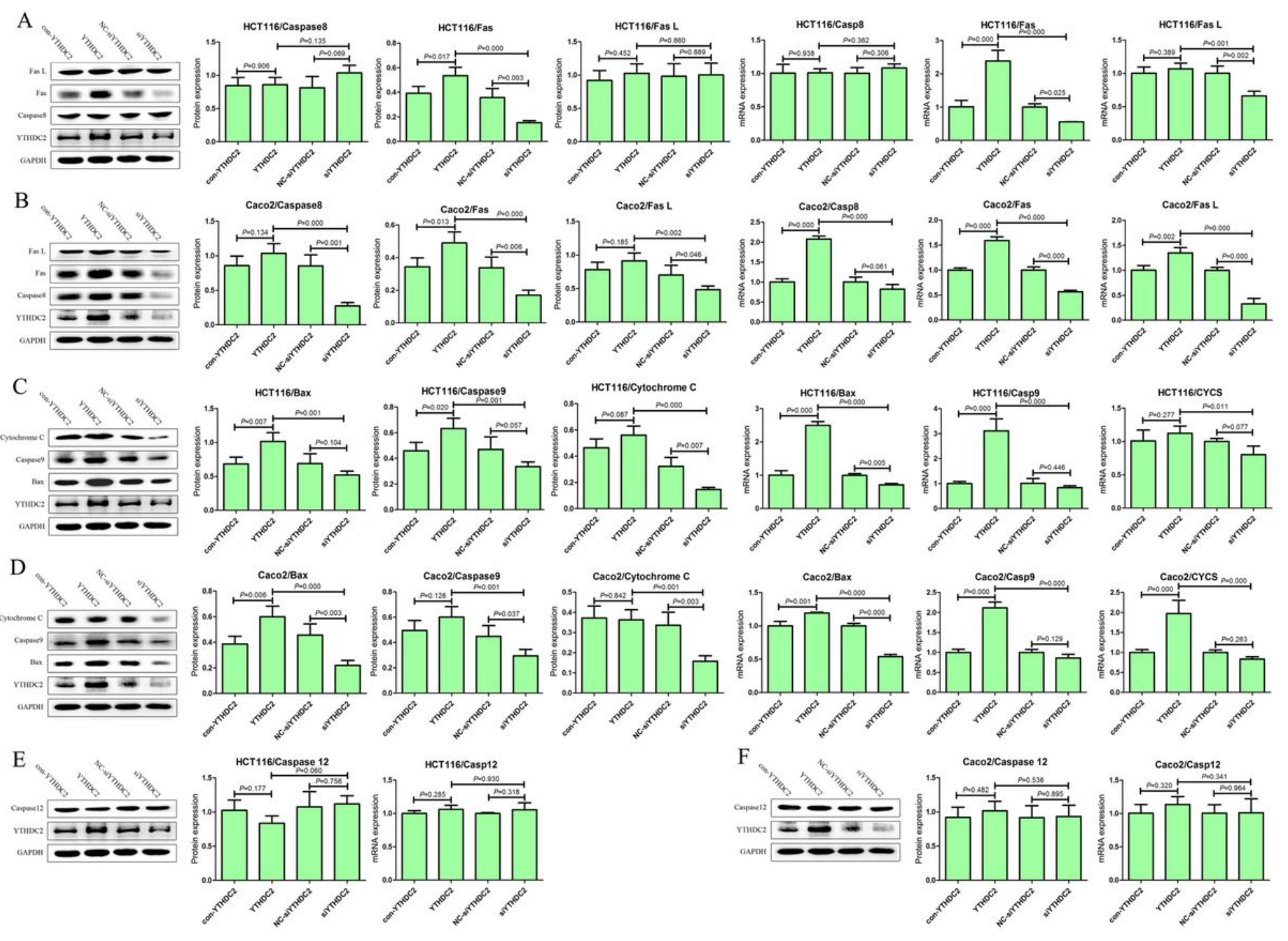

Figure 4

The influence of YTHDC2 on apoptosis-related proteins of the mitogen-associated protein kinase signaling pathway. A) Changes in the expression of apoptotic proteins of the exogenous death receptor activation pathway in HCT116 cells. B) Changes in the expression of apoptotic proteins of the exogenous death receptor activation pathway in Caco2 cells. C) Changes in the expression of apoptotic proteins of the endogenous mitochondrial apoptosis-related pathway in HCT116 cells. D) Changes in the expression of apoptotic proteins of the endogenous mitochondrial apoptosis-related pathway in Caco2 cells. E) Changes in the expression of apoptotic proteins of the endoplasmic reticulum apoptosis pathway in HCT116 cells. F) Changes in the expression of apoptotic proteins of the endoplasmic reticulum apoptosis pathway in Caco2 cells. 